Reprod. Nutr. Dévelop., 1986, 26 (1 B), 129-145.

\title{
Le rendement de la croissance et la biomasse active dans les biotopes anaérobies
}

\author{
J.-P. BELAICH
}

Laboratoire de Chimie bactérienne, C.N.R.S., B.P. 71, 13277 Marseille Cedex 9, France.

Summary. Growth yield and active biomass in anaerobic biotopes.

The molecular growth yield of anaerobic bacteria has been examined in different growth conditions. The influence of kinetics on energy coupling has been discussed.

Les fermentations qui s'opèrent dans les biotopes anaérobies sont réalisées par une multitude de microorganismes qui concourent à la dégradation de la matière organique. Malgré l'énorme travail de microbiologie qui a été réalisé sur ces écosystèmes, dont le rumen est probablement le plus étudié, nous n'avons pas encore la connaissance complète de la composition des flores microbiennes qui les habitent. De nombreux microbes ont cependant pu être isolés et la physiologie et la biochimie d'un petit nombre d'espèces bactériennes, considérées comme représentatives, sont activement étudiées.

L'importance de la biomasse microbienne du rumen dépend de l'efficacité avec laquelle les microorganismes s'y développent. L'importance dans cette biomasse, de chaque espèce particulière dépend également de sa capacité à croître et à se multiplier. Le but de cet article est de discuter des facteurs qui régissent la croissance des microorganismes, et plus particulièrement du rendement de la croissance.

\section{Le rendement de la croissance.}

On doit à Monod d'avoir défini pour la première fois, en 1942, les concepts de rendement énergétique de la croissance et de ration d'entretien, cette dernière notion devant être transformée plus tard en énergie de maintenance. D'innombrables expériences réalisées en milieu non renouvelé ont par la suite conforté les premiers résultats obtenus par Monod à savoir que la biomasse synthétisée est proportionnelle à la quantité d'aliment énergétique utilisé, à condition que ce dernier soit le facteur limitant la croissance.

C'est en 1960 qu'on a évalué le rendement ( $\left.Y_{A T P}\right)$ en fonction de l'énergie utilisable par les systèmes anaboliques: I'ATP. Bauchop et Elsden, étudiant la 
croissance, sur milieu complexe, d'organismes anaérobies dont le catabolisme était assez bien connu pour que l'on puisse calculer la quantité d'ATP produit chaque fois qu'une molécule de substrat énergétique était fermentée, ont montré que la quantité de biomasse synthétisée par de nombreux organismes était d'environ $10,5 \mathrm{~g}$ poids sec, par molécule-gramme d'ATP utilisée. A partir de ces premiers résultats et de nombreuses autres données expérimentales obtenues ultérieurement, qui ont fait l'objet à l'époque des revues de synthèse de Stouthamer (1969), Payne (1970) et Forrest et Walker (1971), il a été conclu que le $Y_{\text {ATP était }}$ une constante biologique. La démonstration paraissait alors assez convaincante pour que certains chercheurs utilisent cette constante pour calculer, à partir des rendements de croissance, la quantité d'ATP générée par les systèmes cataboliques. Hadjipetrou et al. (1964) ont, par exemple calculé un rapport $\mathrm{P} / 0$ de 3 en divisant le rendement $Y_{02}$ de la croissarice aérobie sur glucose de Aerobacter

\section{TABLEAU 1}

$Y_{\text {ATP }}$ de différents organismes se développant en anaérobiose sur des milieux variés.

\begin{tabular}{|c|c|c|c|}
\hline Organisme & Milieu & $Y_{\text {ATP }}$ & Référence \\
\hline Actinomyces israeli & C & 12,3 & Buchanan \& Pine (1967) \\
\hline Actinomyces naeslondii & $\mathrm{C}$ & 18,0 & Buchanan \& Fine (1967) \\
\hline Aerobacter aerogenes & M & 10,2 & Hadjipetrou et al. (1964) \\
\hline Aerobacter cloacae & $\mathrm{M}$ & 11,9 & Hernandez \& Johnson (1967) \\
\hline Anaerovibrio lipolytica & C & 11,0 & Hobson (1965) \\
\hline Bacteroides fragilis & C & 19,6 & Macy et al. $(1975)$ \\
\hline Bacteroides ruminicola & C & 23,0 & Howlett et al. (1976) \\
\hline Bifidobacterium bifidum & C & 13,1 & de Vries et Stouthamer (1968) \\
\hline Clostridium perfringens & C & 14,6 & Hasan \& Hall (1975) \\
\hline Escherichia coli & $\mathrm{M}$ & 11,2 & Stouthamer (1969) \\
\hline Lactobacillus casei & C & 20,9 & de Vries et al. (1970) \\
\hline Lactobacillus plantarum & C & 10,2 & Oxenburgh \& Snoswell (1965) \\
\hline Proteus mirabilis & $\begin{array}{l}\mathrm{C} \\
\mathrm{M}\end{array}$ & $\begin{array}{r}12,6 \\
5,5\end{array}$ & $\begin{array}{c}\text { Stouthamer \& Bettenhaussen (1972) } \\
\text { ibidem }\end{array}$ \\
\hline Ruminococcus flavefaciens & $\mathrm{C}$ & 10,6 & Hopgood \& Walker (1967) \\
\hline Ruminococcus albus & C & 11,5 & Hungate (1963) \\
\hline Saccharomyces cerevisiae & C & 10,2 & Bauchop \& Elsden (1960) \\
\hline Saccharomyces rosei & C & 11,6 & Bulder (1966) \\
\hline Sarcina ventriculi & $\mathrm{s}$ & 11,7 & Stephenson \& Dawes (1971) \\
\hline Selenomonas ruminanticum & C & 15,5 & Hobson \& Summers (1972) \\
\hline Streptococcus agalactiae & C & 9,3 & Mickelson (1972) \\
\hline Streptococcus faecalis & C & 10,9 & $\begin{array}{l}\text { Bauchop \& Elsden (1960) } \\
\text { Sokatch \& Gunsalus (1957) } \\
\text { Forrest \& Walker (1971) } \\
\text { Beck \& Shugart (1966) }\end{array}$ \\
\hline Streptococcus pyogenes & S & 9,8 & Davis et al. (1968) \\
\hline \multirow{6}{*}{ Zymomonas mobilis } & C & 5,9 & McGill \& Dawes (1971) \\
\hline & C & 8,5 & Bauchop \& Elsden (1960) \\
\hline & & & Belaich \& Senez (1965a) \\
\hline & & & $\begin{array}{l}\text { Dawes et al. }(1966) \\
\text { Forrest }(1967)\end{array}$ \\
\hline & $\mathrm{s}$ & 6,5 & Belaich et al. (1972) \\
\hline & M & 4,7 & ibidem \\
\hline
\end{tabular}

$C$ : milieu complexe contenant généralement de l'extrait de levure ; $\mathrm{S}:$ milieu synthétique complet ; $M$ : milieu minimal. 
aerogenes trouvé expérimentalement égal à $62,8 \mathrm{~g}$ par le valeur du $\mathrm{Y}_{\text {ATP }}(10,5)$. Ils en ont conclu que cette bactérie effectuait la phosphorylation oxydative avec la même efficacité que les mitochondries. A partir des années 1970, un certain nombre d'auteurs publiaient des résultats qui n'étaient pas en accord avec la cons-

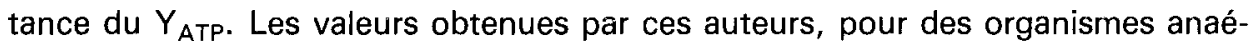
robies se développant sur les milieux divers, variaient de 4,7 pour Zymomonas mobilis (Belaich et al., (1972) à 20,9 pour Lactobacillus casei (de Vries et al., 1970). L'ensemble des données expérimentales est rapporté sur le tableau 1. II devenait alors évident que l'utilisation de 10,5 comme valeur de $Y_{\text {ATP }}$ pour le calcul de la production d'ATP par les systèmes cataboliques pouvait conduire à des résultats erronés et qu'il fallait abandonner I'hypothèse séduisante et unificatrice que tous les microorganismes utilisaient I'ATP, pour leur croissance, avec la même efficacité.

\section{Le $Y_{\text {ATP }}$ théorique.}

On entend par $Y_{\text {ATP }}$ théorique le rendement maximum de croissance qui serait obtenu si tout I'ATP était utilisé pour les biosynthèses. Le calcul de cette

TABLEAU 2

Quantité d'ATP nécessaire à la synthèse des macromolécules.

\begin{tabular}{|c|c|c|c|c|}
\hline Polymère & $\begin{array}{l}\text { Précurseur(s) } \\
\text { contenu } \\
\text { dans le milieu } \\
\text { de culture }\end{array}$ & $\begin{array}{c}\text { Nature } \\
\text { du monomère } \\
\text { et } \mathrm{PM}^{*} \text { moyen }\end{array}$ & $\begin{array}{l}\text { Quantité d'ATP } \\
\text { nécessaire } \\
\text { pour la synthèse } \\
\text { et la polymérisation } \\
\text { du monomère } \\
\text { (Mole d'ATP/Mole } \\
\text { de Monomère) }\end{array}$ & $\begin{array}{l}\text { Quantité d'ATP } \\
\text { nécessaire } \\
\text { à la synthèse } \\
\text { de } 100 \mathrm{~g} \\
\text { de polymère } \\
\text { (Mole) }\end{array}$ \\
\hline Protéine & Amino acide & $\begin{array}{c}\text { Amino acide } \\
110\end{array}$ & 4 & 3,63 \\
\hline ARN & $\begin{array}{c}\text { Bases } \\
\text { Ribose } \\
\text { Phosphate }\end{array}$ & $\begin{array}{c}\text { Nucléoside } \\
\text { triphosphate } \\
300\end{array}$ & 5 & 1,66 \\
\hline ADN & $\begin{array}{c}\text { Bases } \\
\text { Ribose } \\
\text { Phosphate }\end{array}$ & $\begin{array}{c}\text { Déoxynucléoside } \\
\text { triphosphate } \\
280\end{array}$ & 6 & 2,14 \\
\hline $\begin{array}{l}\text { Phospho- } \\
\text { lipides }\end{array}$ & $\begin{array}{c}\text { Glucose } \\
\text { éthanolamine } \\
\text { Phosphate }\end{array}$ & $\begin{array}{c}\text { Phosphatidyl } \\
\text { éthanolamine } \\
\text { (2 palmitates) } \\
675\end{array}$ & 1 & 0,14 \\
\hline $\begin{array}{l}\text { Phospho- } \\
\text { lipides }\end{array}$ & $\begin{array}{c}\text { Acétate } \\
\text { Glycérol } \\
\text { Ethanolamine } \\
\text { Phosphate }\end{array}$ & $\begin{array}{c}\text { Phosphatidyl } \\
\text { éthanolamine } \\
\text { (2 palmitates) } \\
675\end{array}$ & 33 & 4,88 \\
\hline $\begin{array}{l}\text { Polysac- } \\
\text { charides }\end{array}$ & Glucose & $\begin{array}{l}\text { Glucose } \\
162\end{array}$ & 2 & 1,23 \\
\hline Mureine & Glucose & $\begin{array}{l}\text { NAG-NAM } \\
\text { Peptide } \\
1000\end{array}$ & 14 & 1,40 \\
\hline
\end{tabular}


grandeur peut alors se faire facilement grâce à la connaissance, maintenant précise, de la quantité d'ATP nécessaire à la polymérisation des macromolécules biologiques. Le tableau 2 montre la quantité d'ATP dépensée pour la polymérisation de chaque monomère. Il a été réalisé à partir des données publiées par Gunsalus et Shuster (1961), Forrest et Walker (1971), Stouthamer (1973) et Rittenberg et Hespell (1975). La synthèse des protéines demande 4 molécules d'ATP pour la formation de chaque liaison peptide. Le calcul de l'énergie nécessaire à la synthèse de l'ARN a été réalisé en utilisant la séquence classique suivante :

Ribose $5 \mathrm{p}+$ ATP $\rightarrow$ Phosphoribosyl pyrophosphate + AMP

Base (purique ou pyrimidique) + Phosphoribosyl pyrophophaste $\rightarrow$ Nucléoside monophosphate + pyrophosphate

Cette séquence de réactions montre que 3 ATP sont nécessaires pour la formation du phosphoribosyl pyrophosphate et que 2 ATP seront utilisées pour la conversion du nucléoside monophosphate en nucléoside triphosphate, portant ainsi à la 5 la quantité d'ATP utilisée pour l'incorporation d'un nucléotide dans I'ARN. L'incorporation d'un désoxyribonucléotide dans I'ADN nécessite un ATP supplémentaire pour la conversion de chaque nucléotide triphosphate en désoxyribonucléique triphosphate par la ribonucléotide triphosphate réductase et la nucléotide-kinase appropriée. La quantité d'ATP nécessaire à la formation d'un phospholipide est différente suivant que la synthèse s'effectue à partir de l'acétate ou du glucose. L'exemple classique de la synthèse de la phosphatidyl éthanolamine, contenant deux palmitates comme acides gras, permet de calculer que 33 moles d'ATP sont utilisées pour la formation d'une mole de ce composé à partir de l'acétate. En effet la formation de la molécule de glycérol phosphate à partir du glucose demande 1 ATP ; 2 ATP sont nécessaires pour la synthèse du phosphatidyl glycérol pour la rephosphorylation du CMP en CTP et la synthèse de chaque Mole de palmitate à partir de l'acétate exige 8 ATP pour la formation des 8 acétyl CoA et 7 ATP pour la transformation des acétyl CoA en palmityl CoA. La synthèse du même phospholipide à partir du glucose n'exige qu'une seule Mole d'ATP. En effet, pratiquement toutes les bactéries sont capables de transformer le glucose en acétyl CoA avec un gain de 1 ATP par mole d'acétyl CoA et la synthèse des 2 palmitates s'accompagne alors d'un gain net de 2 moles d'ATP. La synthèse des polysaccharides nécessite 2 ATP par liaison glycosidique créée. Enfin la synthèse et la polymérisation du monomère de la mureine, comprenant la disaccharide constitué par la $N$ acétyl glucosamine (NAG) liée à l'acide $N$ acétyl muramique (NAM) et à la fraction peptidique, demande environ 14 ATP. II faut en effet 3 ATP pour la recharge en UTP de l'UMP et de l'UDP relargués après le transfert, respectivement du NAM et du NAG, sur le bactoprenol. Deux ATP sont nécessaires à la formation des 2 fructoses phosphate utilisés pour la synthèse de la glucosamine phosphate et 2 acétyls CoA sont exigés pour la formation de la $\mathrm{N}$ acétylglucosamine. Enfin 5 ATP sont demandés pour la synthèse du pentapeptide du nucléotide de Parks et, si I'on admet que le peptide pont qui relie les tétrapeptides accrochés à la NAM comporte en moyenne 5 amino-acides, [(GLY) 5 par exemple], il faut environ 5 ATP supplémentaires pour la synthèse de ce dernier. On voit que la synthèse du monomère de la mureine demande 17 ATP lorsque l'acétylation des sucres aminés se fait à partir de l'acétate et 13 ATP dans le cas 
où l'acétyl CoA provient du glucose, puisque, dans ce dernier cas, la formation de l'acétyl CoA génère 1 ATP.

Le tableau 3 montre les résultats du calcul du $Y_{\text {ATP }}$ théorique des bactéries lorsque tous les monomères sont fournis par le milieu de culture. On voit que, et ce aussi bien pour les gram ${ }^{+}$que pour les gram $^{-}, c^{\prime}$ est la synthèse des protéines qui nécessite la plus grande quantité d'ATP. Le $Y_{\text {ATP }}$ théorique est approximativement identique pour les 2 catégories d'organismes et, malgré l'amélioration des méthodes d'analyse, en très bon accord avec les premiers résultats de Gunsalus et Shuster (1961) qui ont publié un $Y_{\text {ATP }}$ de 33,3. Les $Y_{\text {ATP }}$ théoriques sont plus forts lorsque le calcul est réalisé en supposant que les phospholipides sont synthétisés à partir du glucose qu'en prenant l'acétate comme source de carbone. Cependant l'écart entre les valeurs obtenues dans les 2 conditions (voir tabl. 3), bien que significatif ( $9 \%$ pour les gram ${ }^{+}$et $15 \%$ pour les gram ${ }^{-}$) ne modifie pas I'ordre de grandeur du $Y_{\text {ATP }}$ théorique. Hespell et Bryant (1979) ont calculé les $Y_{\text {ATP }}$ théoriques de Bdellovibrio bacteriovorus, Megasphera elsdenii et d'une culture mixte, non définie provenant du rumen; les valeurs obtenues pour ces 3 exemples sont respectivement 27,$1 ; 31,9$ et 32,1 ; en bon accord, et cela était prévisible puisque les mécanismes de synthèse sont les mêmes pour tous les organismes vivants, avec les données théoriques calculées pour les gram ${ }^{+}$et les gram $^{-}$.

\section{TABLEAU 3}

Calcul du $Y_{\text {ATP }}$

ATP nécessaire à la synthèse de $100 \mathrm{~g}$ de bactéries/poids sec lorsque les monomères sont fournis à la bactérie

\begin{tabular}{|c|c|c|c|c|}
\hline \multirow[b]{2}{*}{ Polymère } & \multicolumn{2}{|c|}{$\mathrm{Gram}^{+}$} & \multicolumn{2}{|c|}{ Gram $^{-}$} \\
\hline & $\begin{array}{l}\text { Quantité } \\
\text { pour } 100 \mathrm{~g} \\
\text { de bactéries }\end{array}$ & $\begin{array}{l}\text { ATP nécessaire } \\
\text { à la synthèse } \\
\text { (en Mole) }\end{array}$ & $\begin{array}{l}\text { Quantité } \\
\text { pour } 100 \mathrm{~g} \\
\text { de bactéries }\end{array}$ & $\begin{array}{l}\text { ATP nécessaire } \\
\text { à la synthèse } \\
\text { (en Mole) }\end{array}$ \\
\hline Protéine & 60,0 & 2,18 & 52,4 & 1,90 \\
\hline ARN & 15,0 & 0,25 & 16,0 & 0,26 \\
\hline ADN & 4,0 & 0,08 & 3,2 & 0,07 \\
\hline \multirow[t]{2}{*}{ Phospholipides } & 6,0 & $0,29^{a}$ & 9,4 & $0,46^{\mathrm{a}}$ \\
\hline & & $0,008^{b}$ & & $0,01^{\mathrm{b}}$ \\
\hline Polysaccharides & 5,0 & 0,06 & 15 & 0.18 \\
\hline Mureine & 10,0 & 0,14 & 1,6 & 0,02 \\
\hline \multirow[t]{2}{*}{ Total } & & $3,00^{\mathrm{a}}$ & & $2,89^{a}$ \\
\hline & & $2,72^{b}$ & & $2,44^{b}$ \\
\hline \multirow[t]{2}{*}{$Y_{\text {ATP }}$} & & $33,33^{\mathrm{a}}$ & & $34,60^{\mathrm{a}}$ \\
\hline & & $36,76^{b}$ & & 40,98 \\
\hline
\end{tabular}

a : Calcuis effectués en prenant l'acétate comme précurseur de l'acetyl CoA dans la synthèse des phospholipides.

b : Calculs effectués en prenant le glucose comme précurseur de l'acetyl CoA dans la synthèse des phospholipides. On a utilisé les données de Forrest et Walker (1971) pour la composition des bactéries gram $^{+}$et celle de Stouthamer (1973) pour la composition des gram $^{-}$. 
Quelle est la valeur du $\mathrm{Y}_{\text {ATP }}$ théorique lorsque les bactéries se développent dans un milieu minimal contenant du glucose comme source de carbone et d'énergie et une source d'azote simple comme le chlorure d'ammonium ? Stouthamer (1973) a calculé cette grandeur. Le tableau 4 a été réalisé en utilisant une

\section{TABLEAU 4}

$Y_{\text {ATP }}$ théorique de la croissance sur milieu minimal contenant un sucre et du chlorure d'ammonium.

ATP en Mole utilisé pour la synthèse de $100 \mathrm{~g}$ poids sec de bactéries

\begin{tabular}{lc} 
Macromolécule & \\
Protéine & \\
$\quad \begin{array}{l}\text { synthèse des amino acides } \\
\text { polymérisation }\end{array}$ & 0,14 \\
ARN & 1,91 \\
$\quad \begin{array}{l}\text { synthèse des nucléosides monophosphates } \\
\text { polymérisation }\end{array}$ & 0,34 \\
ADN & 0,09 \\
$\quad \begin{array}{l}\text { synthèse des deoxynucléosides monophosphates } \\
\text { polymérisation }\end{array}$ & 0,08 \\
$\begin{array}{l}\text { Polysaccharides } \\
\text { synthèse des monomères } \\
\text { polymérisation }\end{array}$ & 0,02 \\
$\begin{array}{l}\text { Lipides } \\
\quad \text { synthèse des phospholipides }\end{array}$ & 0,00 \\
$\begin{array}{l}\text { Muréine } \\
\text { synthèse et polymérisation }\end{array}$ & 0,20 \\
$\quad \begin{array}{l}\text { Total } \\
\text { YATP }=35,60\end{array}$ & 0,01 \\
$\quad$ & 0,02 \\
\cline { 2 - 2 }
\end{tabular}

partie des données de Stouthamer. Ce calcul a été fait pour une bactérie gram ${ }^{-}$ (forte proportion de lipides, faible quantité de polysaccharide). On voit que le $Y_{\text {ATP }}$ théorique calculé $\left(Y_{\text {ATP }}=35,6\right)$ lorsque la bactérie se développe sur milieu minimal est pratiquement équivalent au $Y_{\text {ATP }}$ calculé dans le cas d'une croissance sur milieu complexe. En d'autres termes, lorsque le substrat énergétique est un sucre, l'énergie nécessaire à la synthèse des monomères est à première vue négligeable par rapport à celle qui est exigée pour leur polymérisation. Etant donné les imprécisions dues à la globalité de l'analyse on peut estimer que la quantité d'énergie utilisée pour la synthèse des monomères représente environ 10 à $20 \%$ de l'énergie totale. Lorsque la source de carbone n'est plus un sucre la demande en ATP pour la synthèse des monomères est plus grande et le $Y_{\text {ATP }}$ théorique décroît. C'est ainsi que Stouthamer (1977) a calculé des $Y_{\text {ATP }}$ théoriques de 13,4 ; 15,4 et 10 pour des organismes se développant sur un milieu minimum contenant respectivement du lactate, du malate et de l'acétate comme source de carbone. 


\section{L'énergie de maintenance.}

Comme il a été dit plus haut le concept d'énergie de maintenance a été pour la première fois défini par Monod sous la formulation de ration d'entretien. Selon Monod : " On peut, dans l'énergie absorbée par un organisme en cours de croissance, distinguer deux parties. L'une représente le travail de synthèse, nécessaire à l'élaboration des matériaux de construction de la cellule. Elle est liée à la croissance et s'annule lorsque le taux de croissance est nul. L'autre représente la dépense nécessaire au maintien de la structure de l'organisme, au travail d'organisation " (Monod, Th. Doct. ès Sci., p. 87). Les techniques de croissance en milieu non renouvelé n'ont pas permis à Monod de démontrer la validité de ce concept. Cette démonstration fut faite à partir de 1958 grâce à l'utilisation du chemostat qui autorise l'obtention, pendant de longues périodes, d'états stationnaires où la croissance s'effectue à un taux inférieur au taux maximum. Les premières expériences qui mirent en évidence l'existence de l'énergie de maintenance ont été rapportées par Herbert (1958) qui montra que, dans le cas de cultures limitées par le glucose, la vitesse spécifique de la consommation énergétique I ds

$\left\langle\mathrm{q}_{\mathrm{s}}=\frac{\mathrm{m} \cdot \mathrm{dt}}{\mathrm{m}} \mathrm{m}\right.$ étant la biomasse et $\mathrm{s}$ le substrat énergétique) était bien proportionnelle au taux de croissance $\mu$, (vitesse spécifique de production de biomasse) mais que la droite qui décrivait la variation de $q_{s}$ en fonction de $\mu$ ne s'extrapolait pas à l'intersection des axes et qu'on avait en réalité une fonction de type :

$$
\mathrm{q}_{\mathrm{s}}=\mathrm{a} \mu+\mathrm{b} \text { ( } \mathrm{a} \text { et } \mathrm{b} \text { étant des constantes) }
$$

Cette relation montre que le rendement moléculaire de la croissance sur le substrat $\left(Y_{s}\right)$, qui est égal au rapport $\mu / q_{s}$, dépend du taux de croissance. Pirt en 1965 formula autrement la relation (1) en écrivant que la vitesse spécifique mesurable de consommation de substrat énergétique $\left(q_{s}\right.$ était la somme de 2 vitesses $q_{g}$ et $q_{m}$.

$$
q_{s}=q_{g}+q_{m}
$$

dans cette relation $q_{g}$ représente la part de la consommation de substrat énergétique qui sert aux biosynthèses et $q_{m}$ la part qui sert à la maintenance. Si l'on divise les deux membres de l'équation (2) par le taux de croissance on obtient en réarrangeant :

$$
\frac{1}{Y_{s}}=\frac{q_{g}}{\mu}+\frac{q_{m}}{\mu} \text {. }
$$

Le terme $q_{g} / \mu$ a les dimensions de l'inverse d'un rendement et représente l'inverse du rendement maximum $Y^{\max }$ avec lequel se développeraient les bactéries si la maintenance était égale à zéro. Le terme $q_{m}$ a par la suite été appelé coefficient de maintenance ou encore énergie de maintenance et on utilise maintenant pour sa représentation le sigle $m_{s}$. Utilisant ces conventions l'équation (3) devient :

$$
\frac{1}{Y_{s}}=\frac{1}{Y_{s}^{\text {max }}}+\frac{m_{s}}{\mu} .
$$

La relation (4) est couramment appelée équation de Pirt. Elle prévoit que l'inverse du rendement mesuré, ou encore apparent, est proportionnel à l'inverse du taux 
de croissance, le coefficient de proportionnalité étant l'énergie de maintenance. Dans cette représentation l'intercept sur l'axe des $1 / Y_{s}$ de la droite représente l'inverse du rendement maxium. Par identification on voit que, dans la relation (1) la constante a représente $1 / Y^{\max }$ et la constante $b$ est le coefficient de maintenance $m_{s}$.

Un très grand nombre de résultats expérimentaux démontrent la validité des relations 1 et 4 . L'équation de Pirt (4) est actuellement la plus utilisée pour l'expression des résultats. Dans de nombreux cas, des écarts par rapport aux droites théoriques ont été obtenus et ces écarts ont permis de mettre en évidence des changements de voies métaboliques provoqués par les variations imposées du taux de croissance. Ce phénomène, extrêmement intéressant et important pour le métabolisme des cultures mixtes naturelles sera examiné en détail plus loin. La production d'ATP dépendant du catabolisme Stouthamer et Betthausen (1973) ont proposé d'exprimer la relation de Pirt en fonction des $\mathrm{Y}_{\text {ATP }}$ suivant la relation :

$$
\frac{1}{Y_{A T P}^{\exp }}=\frac{1}{Y_{\Lambda T P}^{\min }}+\frac{m_{c}}{\mu}
$$

dans cette relation $Y_{A \uparrow p}^{\operatorname{cxp}}$ représente la valeur mesurée, et $m_{e}$ le coefficient de maintenance exprimé en Mole d'ATP par unité de temps et par gramme, poids sec, de microorganismes.

L'utilisation de la relation (5) permet de linéariser la plupart des résultats expérimentaux puisque les données utilisées dans cette équation ne sont plus dépendantes des variations de voies métaboliques. On a rapporté sur le tableau 5 les résultats obtenus pour quelques organismes anaérobies se développant en anaérobiose sans accepteur exogène d'électrons. Ces résultats montrent que le coefficient de maintenance est très variable suivant la nature de l'organisme étudié et, pour un même organisme (cas de Enterobacter aerogenes), suivant la nature du facteur limitant la croissance.

TABLEAU 5

Rendement et coefficient de maintenance d'organismes se développant en anaérobiose.

\begin{tabular}{|c|c|c|c|c|}
\hline Organismes & $\begin{array}{l}\text { Facteur limitant } \\
\text { la croissance }\end{array}$ & $Y_{A T P}^{\max }$ & $\frac{m e}{\text { mole ATP } g^{-1} H^{-1}}$ & Références \\
\hline Candida parapsilopsis & glucose & 12,5 & 0,21 & Rogers et Stewart, 1974 \\
\hline \multirow[t]{4}{*}{ Enterobacter aerogenes } & glucose $M$ & 14,0 & 6,8 & $\begin{array}{l}\text { Stouthamer \& Bettenhaus- } \\
\text { sen, } 1973\end{array}$ \\
\hline & glucose C & 19,9 & 9,9 & Stouthamer, 1975 \\
\hline & tryptophan & 25.4 & 38,7 & Stouthamer, 1977 \\
\hline & citrate & 9,0 & 2,2 & ibidem \\
\hline Escherichia coli & glucose & 10,3 & 18,9 & Hempfling et Mainzer, 1975 \\
\hline Lactobacillus casei & glucose & 24,3 & 1,5 & de Vries et al., 1970 \\
\hline \multirow[t]{2}{*}{ Saccharomyces cerevisiae } & glucose & 11,0 & 0,5 & Watson, 1970 \\
\hline & glucose & 13,0 & 0,25 & Rogers et Stewart, 1974 \\
\hline Saccharomyces cerevisiae & petite & 11,3 & 0,7 & ibidem \\
\hline
\end{tabular}

Le coefficient de maintenance représente la quantité d'énergie qui est utilisée pour des fonctions autres que la croissance, comme par exemple le transport des solutés, la lutte contre les gradients chimiques, la mobilité, l'utilisation de l'infor- 
mation génétique (turn over de l'ARN messager). Stouthamer (1973) a publié une évaluation de la quantité d'énergie nécessaire aux fonctions autres que les biosynthèses. La quantité d'ATP utilisée pour le renouvellement de I'ARN messager, énergie nécessaire à l'utilisation de l'information codée dans le génome, a été calculée à partir des données de Norris et Koch (1972). Ces auteurs ont conclu que, dans des cultures réalisées en milieu non renouvelé, $60 \%$ de l'ARN total synthétisé par le colibacille était du messager. On peut estimer alors la quantité d'ATP utilisée pour la synthèse du messager lors de la production de $100 \mathrm{~g}$ de bactéries poids sec à 0,139 Mole. Cette valeur est beaucoup plus faible que celle qui a été estimée par Forrest et Walker (1971) (1,09 Mole ATP pour $100 \mathrm{~g}$ de cellules) correspondant à une utilisation d'une molécule de messager pour la synthèse de 3 protéines. Plus récemment Hespell et Bryant (1979) ont considéré dans leurs calculs qu'il fallait compter une molécule d'ATP supplémentaire pour la synthèse de la liaison peptide pour comptabiliser l'énergie nécessaire à l'utilisation du code génétique. Cette estimation implique que chaque molécule de messager est utilisée 6 fois. La quantité d'énergie nécessaire au transport des solutés a été évaluée par Stouthamer (1973) à un total de 0,66 Mole d'ATP/100 g de cellules pour le transport des ions ammonium, potassium et phosphate et des amino acides. Les calculs ont été réalisés à partir des revues de Kaback (1972) et de Harold (1972). II

\section{TABLEAU 6}

Rendement $Y_{\text {max. theo. }}$

\begin{tabular}{|c|c|c|c|c|}
\hline & \multicolumn{4}{|c|}{$\begin{array}{l}\text { Mole d'ATP pour la synthèse } \\
\text { de } 100 \mathrm{~g} \text { de bactéries poids sec }\end{array}$} \\
\hline & $\mathrm{Gram}^{+}\left({ }^{1}\right)$ & $\mathrm{Gram}^{-}\left({ }^{1}\right)$ & & $M M\left({ }^{2}\right)$ \\
\hline Synthèse & $\begin{array}{l}3,00^{\mathrm{a}} \\
2,72^{\mathrm{b}}\end{array}$ & $\begin{array}{l}2,89^{\mathrm{a}} \\
2,44^{\mathrm{b}}\end{array}$ & & 2,81 \\
\hline \multicolumn{5}{|l|}{ Energie nécessaire à la synthèse du messager } \\
\hline $\begin{array}{l}\text { 1. Stouthamer (1973) } \\
\text { 2. Forrest et Walker (1971) } \\
\text { 3. Hespell et Bryant (1979) }\end{array}$ & $\begin{array}{l}0,145 \\
1,090 \\
0,545\end{array}$ & $\begin{array}{l}0,139 \\
0,95 \\
0,475\end{array}$ & & $\begin{array}{l}0,139 \\
0,95 \\
0,475\end{array}$ \\
\hline Energie nécessaire au transport des solutés & 0,66 & 0,66 & & 0,66 \\
\hline $\begin{array}{cc}\text { Total } & \text { a1 } \\
& \text { a2 } \\
& \text { a3 } \\
& \text { b1 } \\
& \text { b2 } \\
& \text { b3 }\end{array}$ & $\begin{array}{l}3,80 \\
4,75 \\
4,20 \\
3,52 \\
4,47 \\
3,92\end{array}$ & $\begin{array}{l}3,69 \\
4,5 \\
4,02 \\
3,24 \\
4,05 \\
3,57\end{array}$ & $\begin{array}{l}1 \\
2 \\
3\end{array}$ & $\begin{array}{l}3,61 \\
4,23 \\
3,94\end{array}$ \\
\hline $\begin{array}{r}\text { Ymax theo. } \\
\text { a1 } \\
\text { a2 } \\
\text { a3 } \\
\text { b1 } \\
\text { b2 } \\
\text { b3 }\end{array}$ & $\begin{array}{l}26,28 \\
21,05 \\
23,78 \\
28,38 \\
22,37 \\
25,47\end{array}$ & $\begin{array}{l}27,10 \\
22,22 \\
24,84 \\
30,87 \\
24,69 \\
27,97\end{array}$ & $\begin{array}{l}1 \\
2 \\
3\end{array}$ & $\begin{array}{l}27,7 \\
23,61 \\
25,34\end{array}$ \\
\hline
\end{tabular}

Même convention pour a et $b$ que celle du tableau $3.1,2$ et 3 se rapportent respectivement aux hypothèses de Stouthamer, Forrest et Walker et Hespell et Bryant.

(1) Mêmes conditions de culture que sur le tableau $3 ;\left\langle^{2}\right\rangle$ Mêmes conditions de culture que sur le tableau 4 (milieu minimal). 
est bien évident que d'un point de vue thermodynamique, l'énergie nécessaire à la lutte contre les gradients ioniques dépend de la composition du milieu. Ce point a récemment été discuté par Tempest et Neijssel (1984), il semble que la valeur calculée par Stouthamer (1973) ne doive être considérée que comme un ordre de grandeur. On a calculé sur le tableau 6 les $Y_{\text {ATP }}$ corrigés pour l'énergie qui sert à des fonctions autres que la biosynthèse des macromolécules, cette grandeur sera maintenant appelée Ymax.theo. Les valeurs ont été obtenues à partir des données des tableaux 3 et 4 et des différentes estimations retenues pour l'énergie nécessaire à la synthèse de I'ARN messager. On voit que les $Y$ max.theo. sont nettement inférieurs aux $Y_{\text {ATP }}$ théoriques calculés précédemment et que ces valeurs se rapprochent maintenant des plus grandes valeurs expériementales rapportées (Voir tabl. 1, cas de Lactobacillus casei et Bacteroides ruminicola). C'est la demande en énergie pour la synthèse de l'ARN messager qui est responsable d'une part très importante de l'énergie de maintenance. Des trois hypothèses considérées, celle de Hespell et Bryant semble se rapprocher le plus du vraisemblable. Celle de Stouthamer, correspondant à l'utilisation du messager pour la synthèse de 20 protéines, sous-estime probablement le coût due l'information alors que celle de Forrest et Walker (3 protéines pour un messager) la surestime. Si I'on utilise l'hypothèse d'Hespell et Bryant, la valeur moyenne du Ymax. theo. pour toutes les bactéries s'établit aux environs de 25 . Les calculs montrent que, dans le cas des plus fortes valeurs expériementales de $\mathrm{Y}_{\text {ATP }}$ (Lactobacillus caseï et Bacteroides ruminico/a) le bilan énergétique permet de retrouver $80 \%$ de l'énergie dépensée pour la croissance et la maintenance ce qui, compte tenu de l'insuffisance de données expériementales actuelles représente un résultat honorable. Dans les cas fréquents des $Y_{\text {ATP }}$ expérimentaux compris entre 10-12, seulement $45 \%$ de l'énergie dépensée sont comptabilisés. On doit admettre alors l'existence de processus qui dissipent une partie importante de l'énergie catabolique disponible provoquant ainsi un découplage énergétique de la croissance.

\section{Le découplage énergétique de la croissance.}

Ce sont Rosenberger et Elsden (1960) qui montrèrent clairement que catabolisme et anabolisme pouvaient être, chez les microorganismes, des processus relativement indépendants. Utilisant un chemostat ces auteurs constatèrent que le ralentissement de la croissance de Streptococcus faecalis, provoqué par la carence en tryptophane, n'entrainait pas une diminution corrélative de la vitesse spécifique de dégradation du glucose, substrat énergétique, utilisé dans leurs expériences. En d'autres termes les bactéries faisaient fermenter leur source d'énergie à vitesse constante et indépendante du taux de croissance. Ce phénomène, appelé découplage énergétique, a été observé par de nombreux chercheurs. A la même époque Le Gall et Senez (1960), étudiant la croissance de la bactérie sulfato-réductrice Desulfovibrio desulfuricans, remarquent que le taux et le rendement de la croissance de cette bactérie étaient deux fois moindre en présence d'azote gazeux qu'en présence d'ammonium comme source d'azote alors que la vitesse d'utilisation de la source d'énergie, le lactate, restait constante dans les deux cas. Ces auteurs conclurent en l'existence d'un découplage énergétique quoique le coût énergétique de la réduction de l'azote, non connu à l'époque, 
pouvait expliquer en partie la diminution observée du rendement de la croissance. Belaich (1967) et Belaich et al. (1972) montrent que la croissance de Zymomonas mobilis pouvait être découplée par la carence en acide pantothénique. Dans ces conditions le taux cellulaire d'activité catabolique (fig. 1) est totalement indépen-

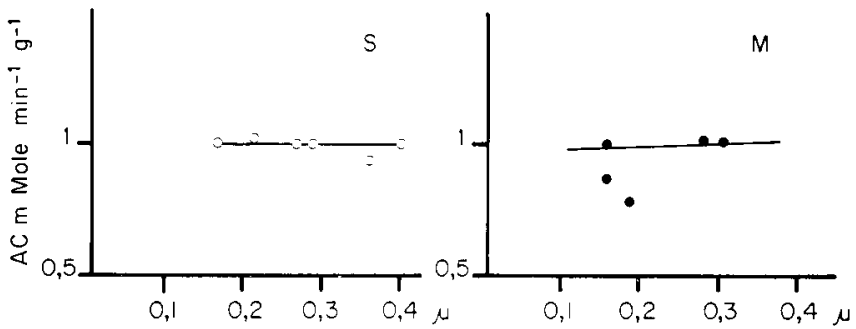

FIG. 1. - Relation entre taux cellulaire d'activité catabolique et taux de croissance chez Zymomonas mobilis.

$S$ : Milieu synthétique contenant tous les monoomères nécessaires à la croissance ; $M$ : Milieu minimal contenant de l'ammoniaque comme seule source d'azote (Belaich, 1967).

dant du taux de croissance. Des expérięnces en chemostat réalisées en 1963 par Neidhart et plus récemment (1976) par Neijssel et Tempest sur la croissance d'Aerobacter aerogenes carencée par un facteur autre que l'énergie comme par exemple le sulfate, le phosphate ou l'ammonium montrent que le catabolisme n'est pas totalement indépendant de I'anabolisme. Cependant on voit sur la figure 2 que le coefficient de maintenance est beaucoup plus important dans le cas où un facteur autre que l'énergie limite la croissance que dans le cas où le glucose

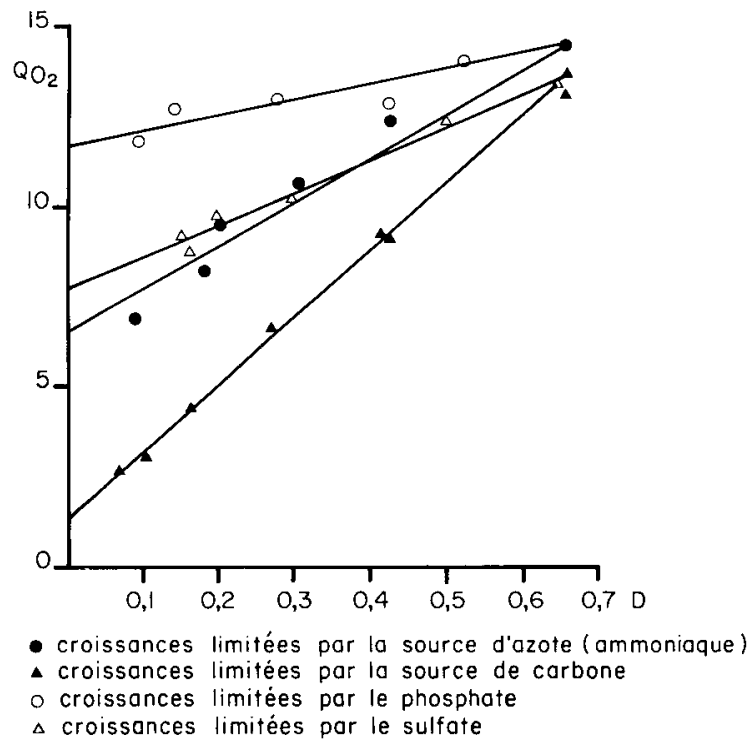

FIG. 2. - Relation entre vitesse spécifique de consommation d'oxygène et taux de croissance chez Klebsielle aerogenes NCTC418 (Neijssel et Tempest, 1976).

$\Delta$ : croissances limitées par la source de carbone; : croissances limitées par la source d'azote (ammoniaque) ; $\triangle$ : croissances limitées par le sulfate ; $O$ : croissances limitées par le phosphate. 
est le facteur limitant. Ces résultats n'invalident pas I'hypothèse du découplage énergétique de la croissance ; ils montrent simplement que le découplage peut être total, comme dans le cas de la croissance de Streptococcus faecalis carencée par le tryptophane (Rosenberger et Elsen, 1960) ou de celle de Zymomonas mobilis limitée par le pantothénate (Belaich, 1967), ou partiel. Belaich (1967), et Belaich et Senez (1965a) ont d'ailleurs montré, en utilisant la microcalorimétrie, que l'arrêt de la croissance de Zymomonas mobilis ou d'Escherichia coli, provoqué par la carence en un facteur autre que l'énergie entraîne un contrôle pouvant aller jusqu'à $50 \%$ de l'activité catabolique des cellules. Ces résultats, sont tout à fait en accord avec ceux de Neidhart (1963) et de Tempest et Neijssel (1976) et renforcent l'idée que le découplage énergétique de la croissance peut, dans certaines situations physiologiques, être partiel et que, corrélativement, un certain contrôle du catabolisme par l'anabolisme peut exister.

L'ensemble des expériences réalisées dans des conditions physiologiques où la source d'énergie n'est pas le facteur limitant met en évidence l'existence d'un découplage total ou partiel de la croissance. Il apparaît donc que, dans ces conditions, une partie plus ou moins importante de l'énergie disponible doit être dissipée. Gunsalus et Shuster (1961) proposaient que l'énergie excédentaire pouvait être dissipée par l'action d'une ATPase directe ou indirecte. L'hypothèse de I'ATPase indirecte a été fréquemment reprise sous la dénomination de cycle futile ; un cycle futile étant constitué par une séquence de réactions conduisant à une hydrolyse gratuite de I'ATP. Ce concept a été très récemment discuté en détail par Tempest et Neijssel (1984). Il est aisé d’identifier, dans le métabolisme, de nombreuses réactions consommatrices d'ATP qui peuvent constituer un cycle futile. Citons par exemple la phosphorylation du fructose 6 phosphate en fructose 1,6 diphosphate suivie de I'hydrolyse du fructose 1,6 diphosphate ou bien l'action de la glutamine synthétase suivie de celle de la glutaminase. Cependant, comme le soulignent Tempest et Neijssel (1984) bien que de nombreux cycles futiles potentiels puissent être reconnus il n'est pas aisé de démontrer leur rôle dans la dissipation de l'énergie excédentaire.

\section{Conclusions.}

La conclusion la plus évidente de toutes les recherches sur l'énergétique de la croissance microbienne est que l'efficacité du couplage énergétique, qui permet l'utilisation par les systèmes anaboliques de l'énergie fournie par les réactions du catabolisme, est étroitement dépendante des conditions cinétiques du développement des microorganismes. II paraît donc opportun de terminer cette revue en examinant, d'une manière détaillée, I'influence des paramètres cinétiques sur la densité microbienne, à l'équilibre, des fermenteurs continus.

La figure 3 montre l'évolution de la biomasse présente dans un chemostat en fonction du taux de dilution et du coefficient de maintenance. Les expériences qui ont permis d'obtenir ces résultats ont été réalisées sur la bactérie sulfatoréductrices Desulfovibrio vulgaris se développant sur lactate comme source d'énergie, en présence de sulfate comme accepteur terminal d'électrons et dans des conditions où le lactate était le facteur limitant la croissance (Traoré et al., 
1983). La manifestation la plus importante de l'existence du coefficient de maintenance est que le réacteur peut être stérilisé par dilution des microorganismes soit dans la région des forts taux de dilution, ce qui est normal, soit dans la région des très faibles taux de dilution et ce phénomène n'est pas " intuitivement " évident. Des résultats expérimentaux identiques ont été publiés dans le cas d'études de la croissance de bactéries du rumen, comme Streptococcus bovis et Selenomonas ruminantium par Hobson (1965) et Hobson et Summers (1972). Ces résultats ont été obtenus dans des conditions de milieux parfaitement homogènes, avec des substrats solubles et des cellules bactériennes non fixées, c'est-à-dire indépendantes les unes des autres. Ces conditions sont fondamentalement différentes de celles qui sont rencontrées dans les biotopes naturels comme les sédiments ou le rumen où I'on se trouve en phase hétérogène avec des bactéries plus ou moins fixées sur des particules. II est donc délicat d'extrapoler sans précaution les résultats obtenus en laboratoire, aux milieux naturels. On peut cependant prévoir que, dans une culture mixte naturelle, une espèce bactérienne donné, placée dans des conditions non saturantes de substrat énergétique, verra sa population obéir à une cinétique proche de celle qui est montrée sur la figure 3.

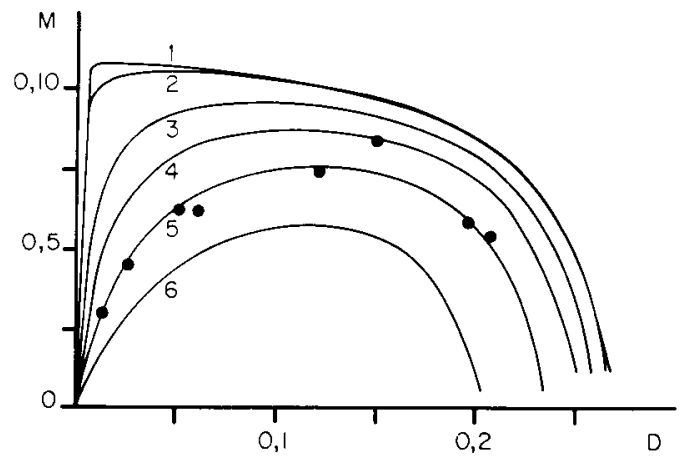

FIG. 3. - Influence du coefficient de maintenance sur la densité microbienne à l'équilibre d'un chemostat.

Les courbes simulées représentent la croissance théorique de la bactérie Desulfovibrio vulgaris se développant en chemostat sur lactate $(14,60 \mathrm{mM})$ sulfate $36 \mathrm{mM}$. Les courbes $1,2,3,4,5,6$ représentent la biomasse à l'équilibre pour des coefficients de maintenance croissance. Les points (•) représentent les données expérimentales (d'après Traoré et a/., 1983).

Chez certains microorganismes les conditions cinétiques de la croissance influent également sur le métabolisme énergétique anaérobie en contrôlant la stœchiométrie des réactions de fermentation. Des changements de voies fermentaires provoqués par un abaissement du taux de croissance peuvent être mis en évidence, en utilisant la relation de Pirt (équation $n^{\circ} 4$ ). On observe alors des écarts sensibles par rapport aux droites théoriques. Un cas exemplaire de contrôle de la stœchiométrie des réactions de fermentation par le taux de croissance a été rapporté par De Vriès et al. (1970) qui ont montré que la bactérie homolactique Lactobacillus casei pouvait, à faible taux de croissance $\left(0,140 \mathrm{H}^{-1}\right)$ opérer la fermentation du glucose sans production de lactate. Dans ces conditions les produits formès sont l'acétate et l'éthanol à raison d'une mole par mole de glucose et le for- 
miate (1,70 Mole par Mole de glucose). Ainsi la fermentation qui était purement lactique à taux maximum de croissance évolue vers une fermentation complètement différente lorsque le taux de croissance imposé en chemostat décroît. Les conséquences d'un tel changement sont importantes. La bactérie synthétise une mole d'ATP supplémentaire grâce à la réaction catalysée par l'acétokinase. De plus, dans le cas des cultures mixtes, la fourniture de produits autres que le lactate comme l'acétate et le formiate peut avoir un effet important sur le développement de certaines espèces bactériennes comme les méthanogènes. D'autres exemples ont été rapportés dans la littérature. Hobson et Summers (1972) ont montré qu'une augmentation de la synthèse d'acétate et de propionate se produisait lorsqu'on diminuait le taux de croissance d'une bactérie lipolytique se développant sur glycérol et que des effets identiques avaient lieu lors de la fermentation du maltose par Bacteroides amylophilus. De même Rosenberger et Elsen (1960) et Hempfling et al. (1969) avaient montré que Streptococcus faecalis produisait, à faible taux de croissance, des acides gras volatils à partir du glucose en quantités beaucoup plus importantes que celles qui étaient synthétisées à fort taux.

Enfin le dernier impact de la cinétique sur l'énergétique de la croissance qu'il convient de discuter brièvement ici est une conséquence directe du métabolisme de l'hydrogène en anaérobiose. C'est en effet le phénomène appelé transfert interspécifique d'hydrogène moléculaire qui autorise la minéralisation anaérobie de la matière organique en méthane et gaz carbonique. Le transfert interspécifique d'hydrogène permet, grâce aux hydrogénases bactériennes, la réoxydattion des pyridine-nucléotides réduites au cours des différentes voies de fermentation en hydrogène moléculaire qui est aussitôt utilisé par les bactéries consommatrices telles que par exemple les bactéries méthanogènes. La conséquence de ce phénomène est que les bactéries donatrices d'hydrogène produisent de l'acétate à la place le l'éthanol et synthétisent des quantités d'ATP plus importantes tout en autorisant la croissance des bactéries méthanogènes. Ce phénomène de transfert d'hydrogène, découvert par Bryant et al. (1967) règle la totalité du métabolisme des cultures mixtes méthanogènes.

Pour terminer cette revue il convient de souligner qu'à ces principaux effets de la cinétique sur la croissance des bactéries s'ajoutent, les phénomènes d'inhibition, de toxicité et d'une manière générale d'antagonismes ou de synergie qui sont encore très mal connus dans les biotopes anaérobies dans lesquels la matière organique est minéralisée. De nombreuses recherches fondamentales sont encore nécessaires pour comprendre convenablement les lois qui régissent l'équilibre des populations microbiennes plurispécifiques responsables de cette action. 


\section{Références}

BAUCHOP T., ELSDEN S. R., 1960. The growth of microorganisms in relation to their energy supply. J. gen. Microbiol., 23, 457-469.

BECK R. W., SHUGART L. R., 1966. Molar growth yields in Streptococcus faecalis vor liquefaciens. J. Bacteriol, 92, 802-803.

BELAICH J. P., 1967. Contribution à l'étude de l'énergétique de la croissance bactérienne. Th. Doct. ès Sci., Univ. d'Aix-Marseille.

BELAICH J. P., SENEZ J. C., 1965a. Application de la méthode microcalorimétrique à l'étude de la croissance bactérienne. Coll. int. CNRS, $\mathrm{n}^{\circ} 156,381-394$.

BELAICH J. P., SENEZ J., 1965b. Influence of aeration and of pantothenate on growth yield of Zymomonas mobilis. J. Bacteriol., 89, 1195-1200.

BELAICH J. P., BELAICH A., SIMONPIETRI P., 1972. Uncoupling in bacterial growth : effect of pantothenate starvation on growth of Zymomonas mobilis. J. gen. Microbiol., 70, 179-185.

BRYANT M. P., WOLIN E. A., WOLIN M. J., WOLFE R. S., 1967. A symbiotic association of two species of bacteria. Arch. Mikrobiol., 59, 29-31.

BUCHANAN B. B., PINE L., 1967. Path of glucose breakdown and cell yields of a facultative anaerobe Actinomyces naes/ondii. J. gen. Microbiol., 46, 225-236.

BULDER C. J. E. A., 1966. Lethality in respiratory deficiency and utilization of fermentation energy in petite negative yeasts. Archiv. Mikrobiol., 53, 189-194.

DAVIS H. C., KARUSH F., RUDD J. H., 1968. Synthesis of M protein by group A hemolytic streptococci in completely synthetic media during steady state growth. J. Bacteriol., 95, 162-168.

DAWES E. A., RIBBONS D. W., REES D. A., 1966. Sucrose utilization by Zymomonas mobilis : formation of a levan. Biochem. J., 98, 804-812.

DE VRIES W., KAPTEIJN W. M. C., BEEK E. G. VAN DER, STOUTHAMER A. H., 1970. Molecular growth vields of Lactobacillus casei L3 in batch cultures and in continuous cultures. J. gen. Microbiol., 63, 333-345.

DE VRIES W., STOUTHAMER A. H., 1968. Fermentation of glucose, lactose, galactose, mannitol and xylose by Bifido bacteria. J. Bacteriol., 96, 472-478.

FORREST W. W., 1967. Energies of activation and uncoupled growth in Streptococcus faecalis and Zymomonas mobilis. J. Bacteriol., 94, 1459-1463.

FORREST W. W., WALKER D. J., 1971. The generation and utilization of energy during growth. Adv. microb. Physiol., 5, 213-274.

GUNSALUS I. C., SHUSTER C. W., 1961. Energy yielding metabolism in bacteria. In I. C. GUNSALUS and R. Y. STANIER, The bacteria, Vol. 2, 1-58, Acad. Press, New-York and London.

HADJIPETROU L. P., GERRITS J. P., TEULINGS F. A. G., STOUTHAMER A. H., 1964. Relation between energy production and growth of Aerobacter aerogenes. J. gen. Microbiol., 36, 139150.

HAROLD F. M., 1972. Conservation and transformation of energy by bacterial membranes. Bacteriol. Rev., 36, 172-230.

HASAN S. M., HALL J. B., 1975. The physiological function of nitrate reduction in Clostridium perfringens. J. gen. Microbiol., 87, 120-128.

HEMPFLING W. P., MAINZER S. E., 1975. Effect of varying the carbon source limiting growth on yield and maintenance characteristics of Escherichia coli in continuous cultures. J. Bacteriol., 123, 1076-1087.

HEMPFLING W. P., MAINZER S. E., VAN DEMARK P. J., 1969. Invariance of $Y$ (adenosine triphosphate) of Streptococcus faecalis $10 \mathrm{cl}$ during anaerobic continuous culture. Bacteriol. Proc., p. 172.

HERBERT D., 1958. Some principles of continuous culture. Symp. int. Congr. Microb., 6, 381-396.

HERNANDEZ E., JOHNSON M. J., 1967. Anaerobic growth yields of Aerobacter clocae and Escherichia coli. J. Bacteriol., 94, 991-995.

HESPELL R. B., BRYANT M. P., 1979. Efficiency of rumen microbial growth : influence of some theoretical and experimental factors on $Y_{\text {ATP. }} J$. anim. Sci. 49, 1640-1659. 
HOBSON P. N., 1965. Continuous culture of some anaerobic and facultatively anaerobic rumen bacteria. J. gen. Microbiol., 38, 167-180.

HOBSON P. N., SUMMERS R., 1972. ATP pool and growth yield in Se/enomonas ruminantium. J. gen. Microbiol., 70, 351-360.

HOPGOOD M. F., WALKER D. J., 1967. Succinic acid production by rumen bacteria. I. Isolation and metabolism of Ruminococcus flavefaciens. Austr. J. biol. Sci., 20, 165-182.

HOWLETT M. R., MOUNTFORT D. O., TURNER K. W., ROBERTSON A. M., 1976. Metabolism and growth yields in Bacteroides ruminicola strain B14. Appl. environ. Microbiol., 32, 274283.

HUNGATE R. E., 1963. Polysaccharide storage and growth efficiency in Ruminococcus albus. J. Bacteriol., 86, 848-854.

KABACK H. R., 1972. Transport ions accross isolated bacterial cytoplasmic membranes. Biochim. biophys. Acta, 265, 367-416.

LE GALL J., SENEZ J. C., 1960. Influence de la fixation de l'azote sur la croissance de Desulfovibrio desulfuricans. C. R. Acad. Sci. Paris, 250, 404-406.

MACY J., PROBST I., GOTTSCHALK G., 1975. Evidence for cytochrome involvement in fumarate reduction and adenosine triphosphate synthesis by Bacteroides fragilis grown in the presence of hemin. J. Bacteriol. Microbiol., 123, 436-442.

McGILL D. J., DAWES E. A., 1971. Glucose and fructose metabolism in Zymomonas anaerobia. Biochem. J., 125, 1059-1068.

MICKELSON M. N., 1972. Glucose degradation, molar growth yields and evidence for oxidative phosphorylation in Streptococcus agalactiae. J. Bacteriol,, 109, 96-105.

MONOD J., 1942. Recherches sur la croissance des cultures bactériennes, Paris, Hermann, $210 \mathrm{p}$.

NEIDHART F. C., 1963. Role of enzyme repression in the regulation of catabolism in bacteria. (Mécanismes de régulation des activités cellulaires chez les microorganismes). Coll. C.N.R.S., 329-336.

NEIJSSEL O. M., TEMPEST D. W., 1976. Bioenergetic aspects of aerobic growth of Klebsiella aerogenes NCT 418 in carbon limited and carbon sufficient chemostat cultures. Arch. Microbiol., 107, 215-221.

NORRIS T. E., KOCH A. L., 1972. Effect of growth rate on the realtive rate of synthesis of messenger, ribosomal and transfer RNA in Escherichia coli. J. mol. Biol., 64, 633-649.

OXENBURGH M. S., SNOSWELL A. M., 1965. Use of molar growth yields in the evaluation of energy producing pathway in Lactobacillus plantarum. J. Bacteriol., 89, 913-914.

PAYNE W. J., 1970. Energy yields and growth of heterotrophs. Ann. Rev. Microbiol,, 24, 17-52.

PIRT S. J., 1965. The maintenance energy of bacteria in growing cultures. Proc. roy. Soc. London, ser, $B, 163,224-231$.

RITTENBERG S. C., HESPELL R. B., 1975. Energy efficiency of intraperiplasmic growth of Bdellavibrio bacteriovorus. J. Bacteriol., 121, 1158-1165.

ROGERS P. J., STEWART P. R., 1974. Energetic efficiency and maintenance energy characteristics of Saccharomyces cerevisiae (wild type and petite) and Candida parapsilosis grown aerobically and microaerobically in continuous cultures. Arch. Microbiol., 99, 25-46.

ROSENBERGER R. F., ELSDEN S. R., 1960. The yields of Streptococcus faecalis grown in continuous culture. J. gen. Microbiol, 22, 726.

SOKATCH J. T., GUNSALUS I. C., 1957. Aldonic acid metabolism. I. Pathway of carbon in an inducible gluconate fermentation by Streptococcus faecalis. J. Bacteriol., 73, 452-460 (à corriger dans le texte).

STEPHENSON M. P., DAWES E. A., 1971. Pyruvic and formic acid metabolism in Sarcina ventriculi and the role of ferredoxin. J. gen. Microbiol., 69, 331-343.

STOUTHAMER A. H., 1969. Determination and significance of molar growth yields. In NORRIS J. R., RIBBONS D. W., Methods in microbiology. Vol. 1, 629-663, Acad. Press, London and New-York.

STOUTHAMER A. H., 1973. A theoretical study of the amount of ATP required for synthesis of microbial cell material. Antonie van Leeuwenhoek, 39. 545-565.

STOUTHAMER A. H., 1977. Energetic aspects of the growth of microorganisms. Symp. Soc. gen. Microb., 27, 285-315.

STOUTHAMER A. H., BETTENHAUSSEN C. W., 1972. Influence of hydrogen acceptor on growth and energy production of Proteus murabilis. Antonie van Leeuwenhoek, 38, 81-90. 
STOU THAMER A. H., BETTENHAUSSEN C. W., 1973. Utilization of energy for growth and maintenance in continuous and batch cultures of microorganisms. A reevaluation of the method of determination of ATP production by measuring the molar growth yields. Biochem. biophys. Acta, 301, 53-70.

STOUTHAMER A. H., BETTENHAUSSEN C. W., 1975. Determination of the efficiency of oxidative phosphorylation in continuous cultures of Aerobacter aerogenes. Arch. Microbiol., 102, 187192.

TEMPEST D. W., NEIJSSEL O. H., 1984. The statues of $Y_{A T P}$ and maintenance energy as biologically interpretable phenomena. Ann. Rev. Microbiol., 38, 459-484.

TRAORE A., GAUDIN C., HATCHIKIAN C. E., LE GALL J., BELAICH J. P., 1983. Energetic of growth of a defined mixed cultures of Desulfovibrio vulgaris and Methanosarcina barkeri : Maintenance energy coefficient of the sulfate reducing organism in the absence and presence of its partner. J. Bacteriol., 155, 1260-1264.

WATSON T. G., 1970. Effect of sodium chloride on steady state growth and metabotism of Saccharomyces cerevisiae. J. gen. Microbiol., 64, 91-99. 